\title{
REVISTAMATËRIA
}

ISSN 1517-7076 artigo e11774, 2017

\section{Correlação entre variáveis térmicas de solidificação, microestrutura e resistência mecânica da liga Al-10\%Si-2\%Cu}

\author{
Correlation between solidification thermal \\ variables, microstructure and mechanical \\ strength of an $\mathrm{Al}-10 \mathrm{wt} \% \mathrm{Si}-2 \mathrm{wt} \% \mathrm{Cu}$ alloy
}

\author{
Maurício Silva Nascimento ${ }^{1}$, Carlos Frajuca ${ }^{1}$, \\ Francisco Yastami Nakamoto ${ }^{1}$, Givanildo Alves dos Santos ${ }^{1}$, \\ Antonio Augusto Couto ${ }^{2,3}$.
}

\footnotetext{
${ }^{1}$ IFSP - Instituto Federal de Educação, Ciência e Tecnologia de São Paulo -São Paulo -Brasil

e-mail: mauricio.nascimento@ifsp.edu.br; frajuca@ifsp.edu.br; nakamoto@ifsp.edu.br; givanildo@ifsp.edu.br

${ }^{2}$ IPEN - Instituto de Pesquisas Energéticas e Nucleares -São Paulo -Brasil

${ }^{3}$ UPM - Universidade Presbiteriana Mackenzie -São Paulo -Brasil

e-mail: acouto@ipen.br
}

\begin{abstract}
RESUMO
As condições operacionais impostas nos processos de fundição geram, como consequência direta, uma diversidade de estruturas de solidificação. Em geral, as propriedades mecânicas mostram-se fortemente dependentes da morfologia estrutural e da composição química. Um aço de baixo carbono foi utilizado para promover fluxo de calor unidirecional durante a solidificação e obter o arranjo da microestrutura. $\mathrm{O}$ objetivo deste trabalho é pesquisar a influência das variáveis térmicas de solidificação na microestrutura da liga Al-10\%Si$2 \% \mathrm{Cu}$ e na sua resistência mecânica. Resultados experimentais incluem os espaçamentos dendríticos primários (EDP), velocidade de deslocamento da isoterma liquidus $\left(\mathrm{V}_{\mathrm{L}}\right)$, taxa de resfriamento $\left(\mathrm{T}_{\mathrm{R}}\right)$ e limites de resistência à tração (LRT) e de escoamento (LE). Estruturas dendríticas mais grosseiras tendem a diminuir a resistência à tração e ao escoamento na liga em estudo.
\end{abstract}

Palavras-chave: solidificação, microestrutura, espaçamentos dendríticos primários, propriedades mecânicas, liga Al-10wt.\%Si-2wt.\%Cu.

\begin{abstract}
The imposition of a wide range of operational conditions in foundry and castings process generates, as a direct consequence, a diversity of solidification structures. The mechanical properties show to depend strongly on the structural morphology and chemical composition. A low carbon steel mold was used to promote a unidirectional heat flow during solidification and to obtain the arrangement of the microstructure. The aim of the present article is to investigate the effect of thermal solidification variables on the microstructure of the Al$10 \mathrm{wt} . \% \mathrm{Si}-2 \mathrm{wt} . \% \mathrm{Cu}$ alloy and its mechanical strength. Experimental results include primary dendrite arm spacings (EDP), tip growth rate $\left(\mathrm{V}_{\mathrm{L}}\right)$, cooling rate $\left(\mathrm{T}_{\mathrm{R}}\right)$ and tensile (LRT) and yield (LE) strength limits. A coarser dendritic structure tends to improve the tensile strength and the yield tensile strength in the studied alloy.
\end{abstract}

Keywords: solidification, microstructure, primary dendrite arm spacings, mechanical properties, Al-10 $\mathrm{wt} \% \mathrm{Si}-2 \mathrm{wt} \% \mathrm{Cu}$ alloy.

\section{INTRODUÇÃO}

Estudos sobre solidificação de ligas multicomponentes em regime transitório são escassos na literatura especializada [1]. O sistema ternário alumínio, silício e cobre possui excelentes propriedades como elevada resistência mecânica, baixo peso e boa fluidez. Essas características fazem desse sistema uma boa escolha na indústria automotiva e aeroespacial chamando a atenção de pesquisadores [2]. O presente trabalho objetiva obter correlações entre microestrutura, variáveis térmicas de solidificação e propriedades mecânicas da liga 
Al-10\% $\mathrm{Si}-2 \% \mathrm{Cu}$, analisando a influência de variáveis térmicas de solidificação sobre a formação microestrutural da liga estudada e desenvolver correlações entre espaçamentos dendríticos primários e resistência mecânica. A partir do controle de variáveis térmicas de solidificação, como a velocidade de deslocamento da isoterma liquidus $\left(V_{L}\right)$ e taxa de resfriamento $(\dot{\mathrm{T}})$, é possível preestabelecer condições para se obter microestruturas que permitam obter propriedades desejadas $[3,4]$.

$\mathrm{O}$ controle da microestrutura durante o processo de solidificação continua a apresentar numerosos desafios científicos e tecnológicos [5] sendo este essencial para qualquer atividade de processamento [6]. O tipo de microestrutura de solidificação que se forma na transição sólido/líquido depende de variáveis como o grau de super-resfriamento, o gradiente térmico à frente da interface sólido/líquido, a velocidade da interface e a concentração de soluto. Uma interface plana, por exemplo, ocorre em situações de baixas velocidades de resfriamento $\left(\mathrm{V}_{\mathrm{L}}\right)$, baixa concentração de soluto $(\mathrm{Co})$, baixo grau de super-refriamento constitucional (SRC) e altas taxas de gradiente térmico $\left(\mathrm{G}_{\mathrm{L}}\right)$, o que torna improvável obter essa estrutura em condições operacionais na maioria das operações metalúrgicas em um sistema metal/molde [7]. A Figura 1 mostra esquematicamente a influência dessas variáveis na microestrutura resultante.

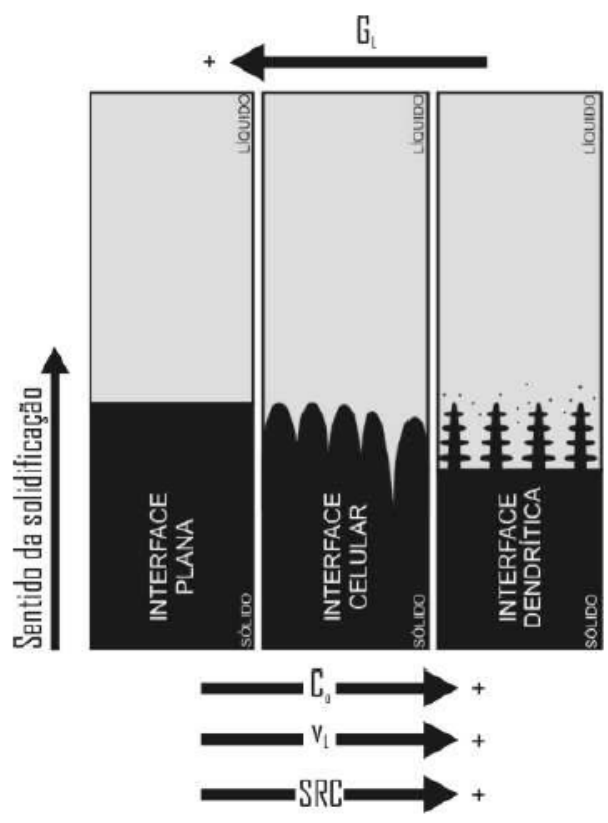

Figura 1: Influência das variáveis de solidificação na microestrutura resultante [8].

Dentre os tipos de microestrutura, a dendrítica é a mais comumente observada durante o processamento de estruturas de solidificação de ligas [9] e ocorre para maiores velocidades de resfriamento e menores gradientes de temperaturas no líquido, consequentemente maior região de super-resfriamento constitucional. Um modo muito utilizado para determinar os efeitos das condições de solidificação sobre a microestrutura dedrítica formada consiste na medida dos espaçamentos dendríticos primários (EDP), secundários (EDS) e dependendo do sistema considerado, os espaçamentos dendríticos terciários (EDT). Esses espaçamentos exercem uma influência significativa sobre as propriedades mecânicas de ligas em seu estado bruto de solidificação. A Figura 2 ilustra como são medidos esses espaçamentos. 


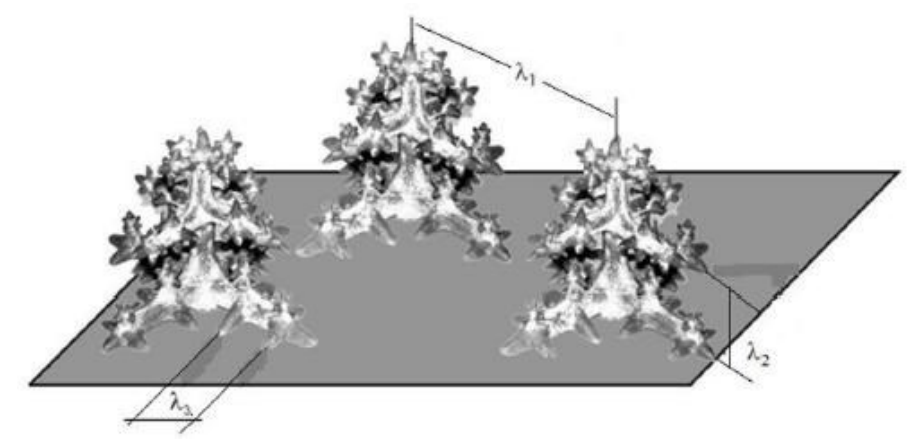

Figura 2: Ilustração do aspecto morfológico de crescimento dendrítico unidirecional em que $\left(\lambda_{1}\right),\left(\lambda_{2}\right)$ e $\left(\lambda_{3}\right)$ são, respectivamente, os espaçamentos primários, secundários e terciários [8].

\section{MATERIAIS E MÉTODOS}

A Figura 3 apresenta um desenho esquemático do forno de solidificação unidirecional utilizado nos experimentos. Um critério importante foi obter fluxo de retirada de calor de forma descendente por meio de solidificação direcional ascendente. As resistências laterais do interior do forno tinham como objetivo aquecer o metal líquido dentro da lingoteira e evitar a perda de calor pelas laterais durante o resfriamento. A lingoteira de aço inoxidável, utilizada no experimento, possuí como dimensões $60 \mathrm{~mm}$ de diâmetro interno, $76 \mathrm{~mm}$ de diâmetro externo e altura de $160 \mathrm{~mm}$. Possui furos laterais nos quais sete termopares do tipo $\mathrm{K}$ foram dispostos em distâncias de $4,8,12,16,35,73,53$ milímetros em relação à chapa (ou molde) de resfriamento. A superfície lateral interna da lingoteira foi revestida com camada refratária de alumina. A extração de calor ocorreu em função dessa chapa de resfriamento, confeccionada em aço ABNT 1010 e espessura de $5 \mathrm{~mm}$, na qual a superfície de contato com o metal líquido foi devidamente polida. A superfície inferior da chapa foi resfriada por um fluxo de água constante de vazão 21,6 1/min.

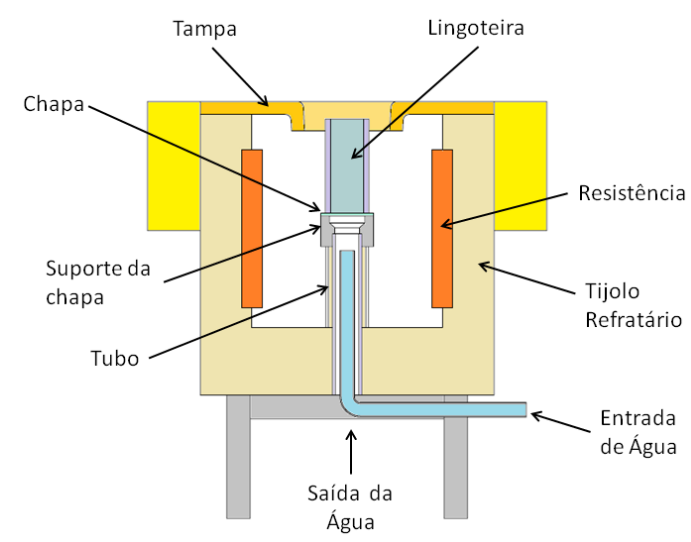

Figura 3: Ilustração esquemática do forno de solidificação unidirecional ascendente.

Todos os termopares foram conectados a um conjunto módulo de aquisição de dados NI 9212 e chassi NI cDAQ 9171 da National Instruments, que enviavam os dados coletados via cabo USB para um computador, permitindo assim a coleta de dados de temperatura em função do tempo, a uma taxa de um dado por segundo. O sistema experimental é mostrado na Figura 4. 


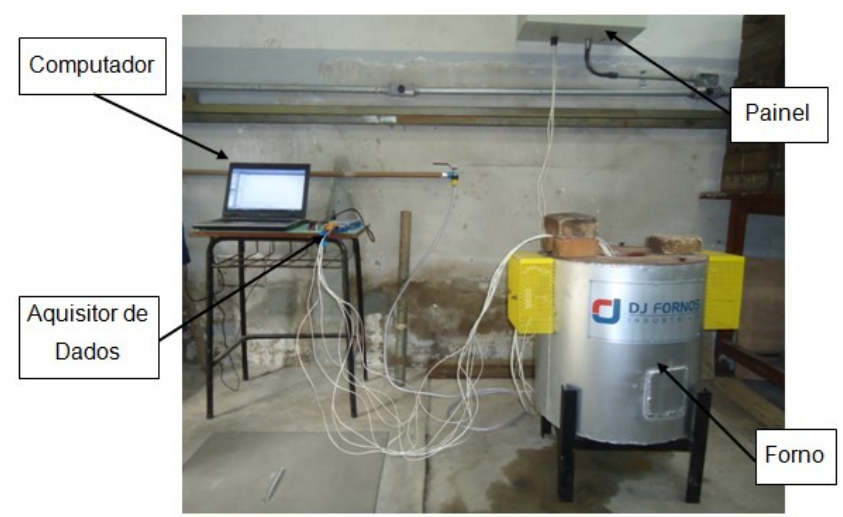

Figura 4: Foto do sistema experimental utilizado no experimento de solidificação.

A liga foi preparada em um cadinho de grafite-argila em forno elétrico do tipo mufla, utilizando-se metais comercialmente puros, os quais foram analisados pela técnica de espectrometria de raios-X, conforme mostra a Tabela 1 .

Tabela 1: Composição química dos materiais em \%peso.

\begin{tabular}{|l|c|c|c|c|c|c|c|c|c|c|c|}
\hline Elemento & Al & Si & Ca & Fe & Ti & Cu & Mg & Zn & NI & Cl & Outros \\
\hline Alumínio & 99,8 & 0,071 & & 0,095 & & & 0,001 & & & & 0,033 \\
\hline Silício & 1,56 & 96,4 & 0,486 & 0,430 & 0,110 & & 0,378 & & & 0,199 & 0,437 \\
\hline Cobre & 1,16 & 0,139 & 0,143 & & & 97,4 & & 0,288 & 0,207 & 0,164 & 0,499 \\
\hline
\end{tabular}

Foram realizados quatro vazamentos, todos realizados com temperatura de vazamento de $700^{\circ} \mathrm{C}$, sendo que o primeiro lingote foi utilizado para análise da cinética de solidificação e os outros três para confeccionar corpos de prova para ensaios de tração. Para cada vazamento foi retirada uma amostra para checar a composição a liga preparada. A análise foi realizada pela técnica de espectrometria de raios-X e os valores médios de composição dos quatros vazamentos são apresentados na Tabela 2.

Tabela 2: Composição química média dos lingotes em \%peso.

\begin{tabular}{|c|c|c|c|c|c|c|}
\hline Elemento & Si & Cu & Fe & Cr & Outros & Al \\
\hline Porcentagem & 9,99 & 1,905 & 0,219 & 0,080 & 0,006 & 87,8 \\
\hline
\end{tabular}

A função $\mathrm{P}=\mathrm{f}(\mathrm{t})$ foi definida experimentalmente pela intersecção da reta equivalente a temperatura liquidus $\left(\mathrm{T}_{\mathrm{L}}\right)$ da liga com a curva de resfriamento de cada termopar. Por meio da dessa intersecção da reta determina-se o tempo de passagem da isoterma liquidus naquela posição de cada termopar. A derivada da função $P=f(t)$ permitiu a obtenção dos respectivos valores experimentais para as velocidades de deslocamento da isoterma liquidus $\left(\mathrm{V}_{\mathrm{L}}\right)$, o que corresponde à passagem da frente líquida por cada termopar. Os valores de taxa de resfriamento $\left(T_{R}\right)$ para cada posição dos termopares foram obtidos experimentalmente a partir das intersecções da reta da temperatura liquidus com as curvas de resfriamento de cada termopar, através do resultado da leitura direta do quociente das temperaturas antes e depois da temperatura liquidus e dos tempos correspondentes, isto é $\Delta \mathrm{T} / \Delta \mathrm{t}$.

Amostras transversais foram retiradas de um dos lingotes solidificados para análise metalográfica nas posições 5, 10, 20, 30, 40, 60 e 70 milímetros em relação a chapa de resfriamento. Estas foram embutidas em baquelite, lixadas e polidas com pasta de diamante de $6 \mu \mathrm{m}$ até $3 \mu \mathrm{m}$. O reagente para ataque utilizado para revelar a microestrutura foi uma solução de $0,5 \%$ HF com um tempo de imersão para reação entre 20 e 25 segundos. Foi utilizada a microscopia óptica para medir os espaçamentos dendríticos primários (EDP) para cada posição selecionada, sendo o método do triângulo o utilizado [10]. Foram realizadas 40 medições para cada posição, sendo realizadas somente na região colunar como forma de correlacionar essa macroestrutura com as variáveis térmicas e resistência a tração.

Os corpos de prova foram confeccionados a partir de três lingotes, proporcionando a realização de en- 
saio de tração de três corpos de prova para cada posição definida conforme Figura 5. A face inferior do lingote é a que esteve em contato com a chapa de troca de calor.

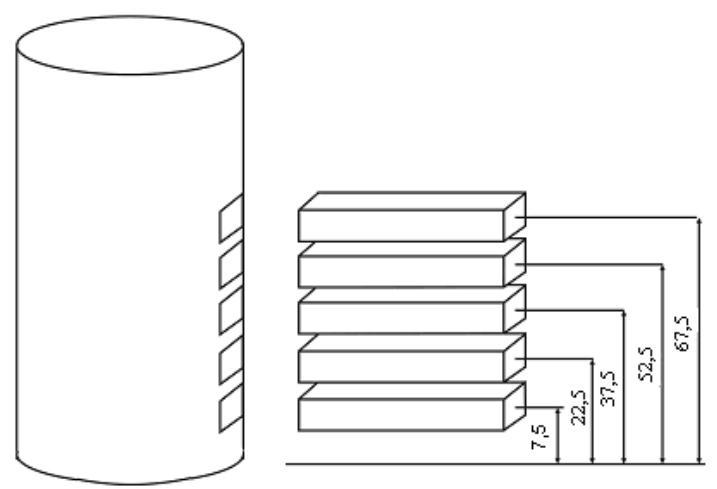

Figura 5: Localização dos corpos de prova para ensaio de tração (dimensões em milímetros).

Os corpos de prova foram confeccionados conforme desenho ilustrativo apresentado na Figura 6. O equipamento utilizado para a realização do ensaio foi uma máquina universal de ensaios Kratos que possui software próprio para armazenamento e apresentação de dados.

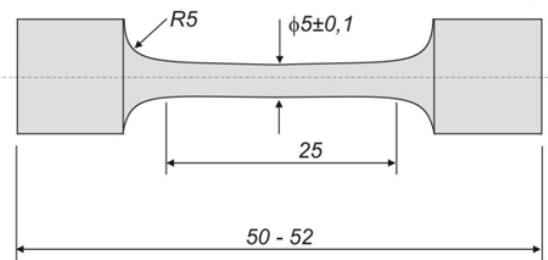

Figura 6: Desenho ilustrativo dos corpos-de-prova conforme previsto nas normas ASTM E 8M e ABNT NBR 6892-1 2013 versão corrigida 2015 (dimensões em milímetros)

\section{RESULTADOS E DISCUSSÃO}

As temperaturas experimentalmente monitoradas para cada termopar são apresentadas na Figura 7. A temperatura liquidus para a liga em estudo foi obtida no diagrama pseudo binário $\mathrm{Al}-10 \% \mathrm{Si}-\mathrm{XCu}$ apresentado na Figura 8.

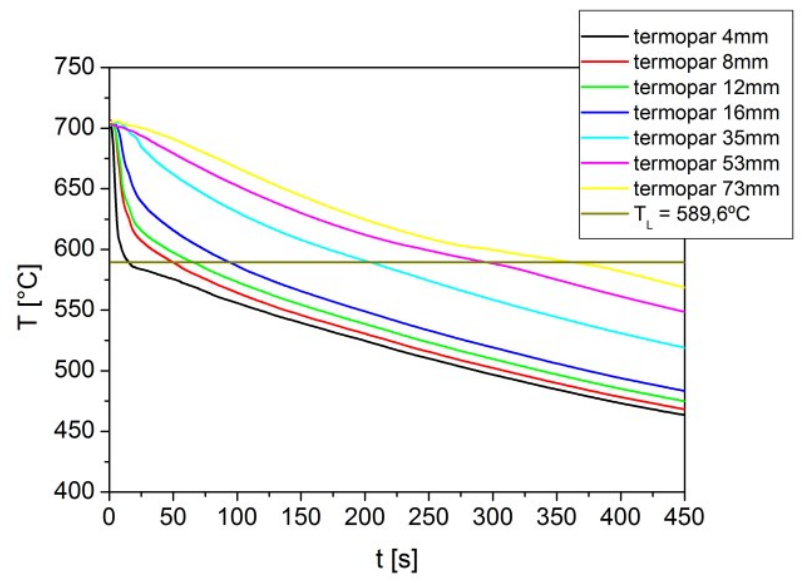

Figura 7: Curvas de resfriamento para posições específicas. 


\section{Diagrama de Equilíbrio Al-10Si-Cu (\%p)}

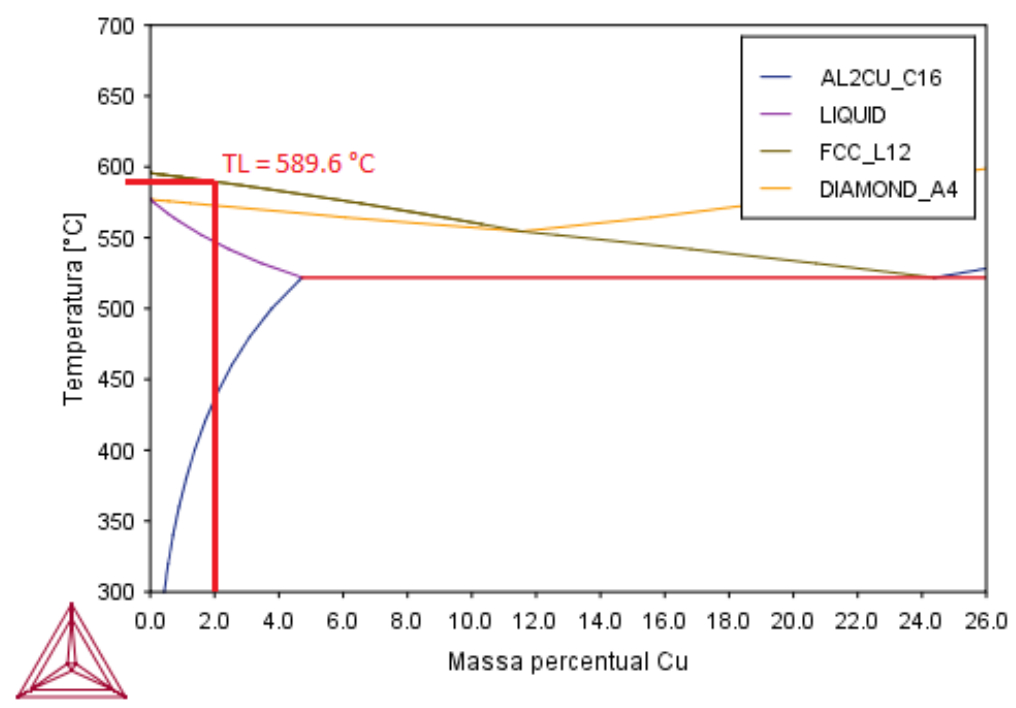

Figura 8: Diagrama pseudo binário parcial da liga $\mathrm{Al}-10 \% \mathrm{Si}-\mathrm{XCu}$.

As Figuras 9 e 10 apresentam, respectivamente, a velocidades de deslocamento da isoterma liquidus $\left(V_{L}\right)$ e a taxa de resfriamento $\left(T_{R}\right)$, ambas em função da posição em relação à interface metal/molde. Pode-se observar que tanto a $\mathrm{V}_{\mathrm{L}}$ como a $\mathrm{T}_{\mathrm{R}}$ diminuem com o aumento dos valores de posição. Tal fato pode ser explicado pela elevação da resistência térmica à extração de calor por condução pelo aumento da camada solidificada [11].

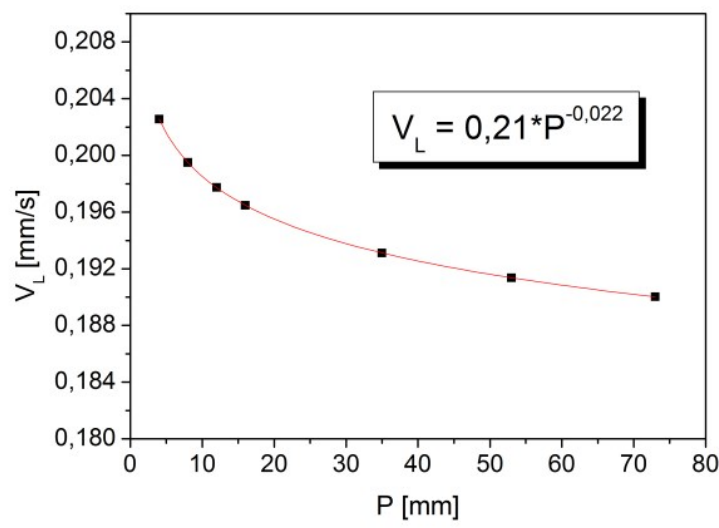

Figura 9: Relação entre velocidade da isoterma liquidus $\left(\mathrm{V}_{\mathrm{L}}\right)$ em função da posição $(\mathrm{P})$. 


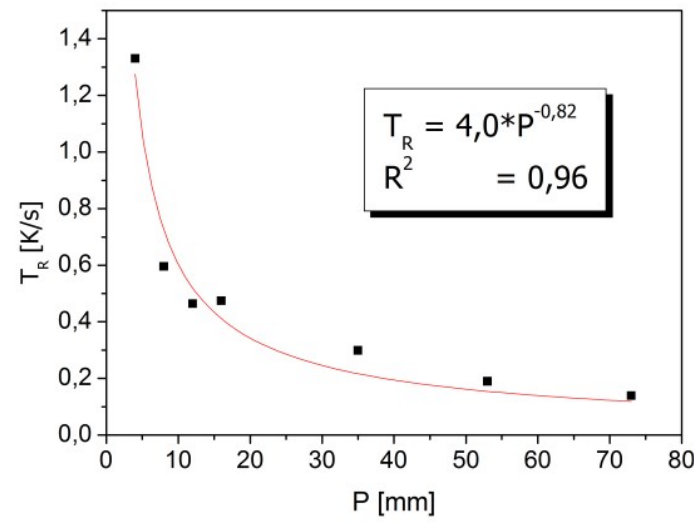

Figura 10: Relação entre taxa de resfriamento $\left(T_{R}\right)$ em função da posição $(P)$.

A Figura 11 apresenta a macrografia de um lingote fundido evidenciando a estrutura colunar obtida na solidificação. Esse mesmo procedimento foi realizado para os outros lingotes, que foram cortados posteriormente para confecção dos corpos de prova.

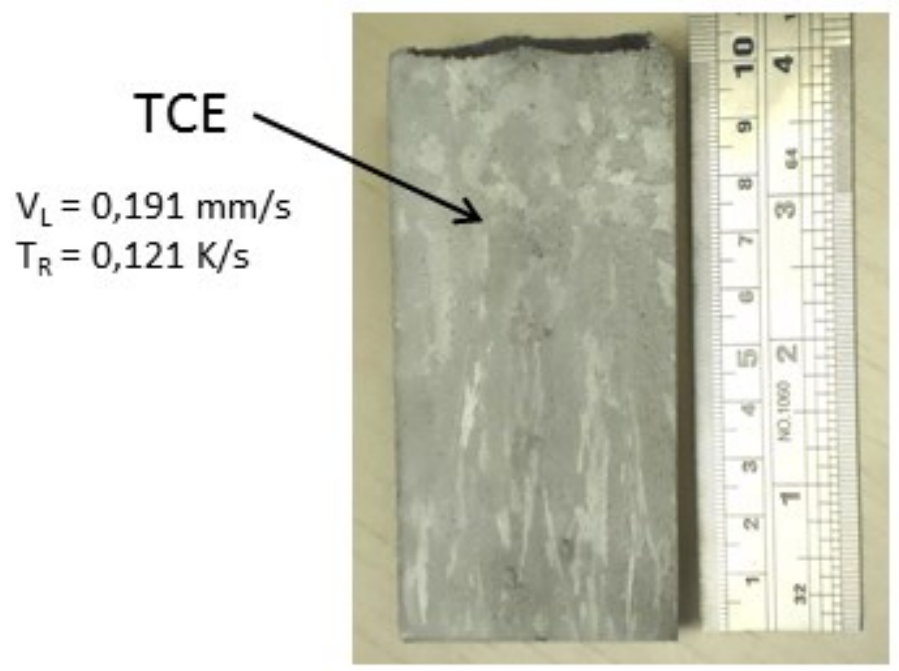

Figura 11: Imagem da macrografia do lingote resfriado evidenciando a estrutura colunar, a transição colunar-equiaxial (TCE) e a estrutura equiaxial. Os valores de $V_{L}$ e $T_{R}$ para a TCE são apresentados na figura.

A Figura 12 apresenta microestruturas transversais típicas da liga em diferentes posições em relação á interface metal/molde. 


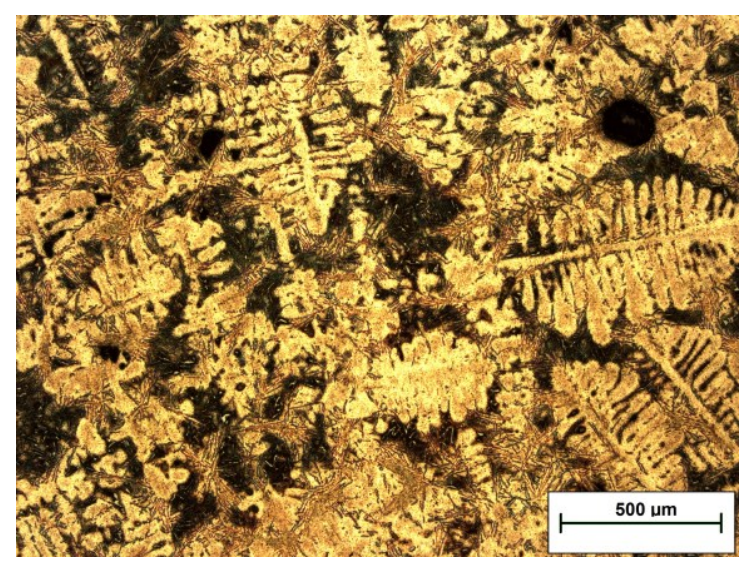

$$
\begin{aligned}
& \text { (Posição } 10 \mathrm{~mm} \text { ) } \\
& \mathrm{V}_{\mathrm{L}}=0,198 \mathrm{~mm} / \mathrm{s} \\
& \mathrm{T}_{\mathrm{R}}=0,604 \mathrm{~K} / \mathrm{s} \\
& \mathrm{EDP}=538 \mu \mathrm{m}
\end{aligned}
$$

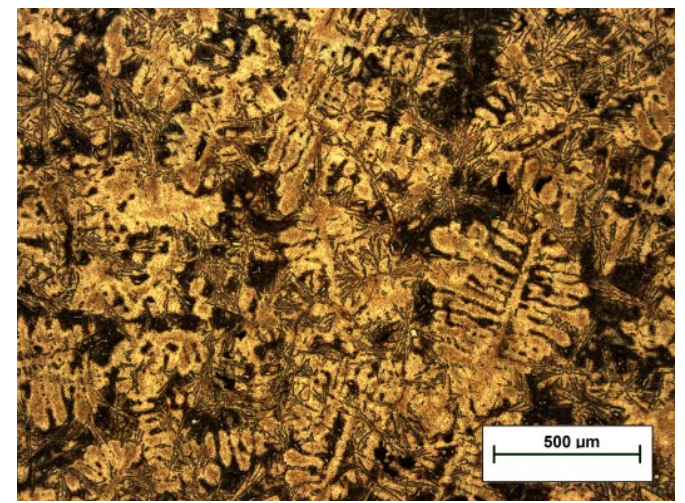

$$
\begin{array}{cc}
\text { (Posição } 30 \mathrm{~mm} \text { ) } & \text { (Posição } 60 \mathrm{~mm} \text { ) } \\
\mathrm{V}_{\mathrm{L}}=0,194 \mathrm{~mm} / \mathrm{s} & \mathrm{V}_{\mathrm{L}}=0,191 \mathrm{~mm} / \mathrm{s} \\
\mathrm{T}_{\mathrm{R}}=0,246 \mathrm{~K} / \mathrm{s} \ldots \ldots \ldots \ldots \ldots \ldots \ldots \ldots \ldots \ldots \ldots \ldots \ldots \ldots . . . \cdots \\
\mathrm{E}
\end{array}
$$

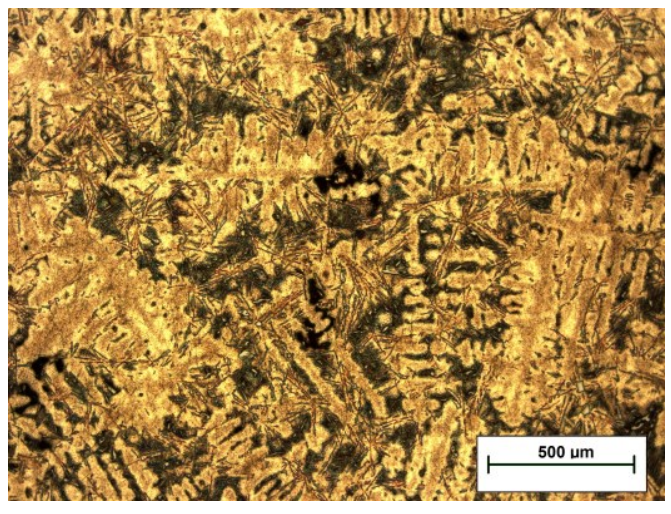

Figura 12: Micrografias da liga $\mathrm{Al}-10 \% \mathrm{Si}-2 \% \mathrm{Cu}$ e respectivas posições relativas, com o mesmo aumento óptico (50 vezes), evidenciando o refino do espaçamento dendrítico primário em posições mais próximas da interface metal/molde (barra de medida com $500 \mu \mathrm{m}$ ).

A Figura 13 apresenta a equação experimental que correlaciona os espaçamentos dendríticos primários e a posição relativa à interface metal/molde, de onde se obteve a lei experimental. As barras de erro foram definidas como o desvio padrão das 40 medidas realizadas utilizando o método do triângulo [10]. Foram obtidos dados de EDP na região colunar, portanto até uma distância de $70 \mathrm{~mm}$. 


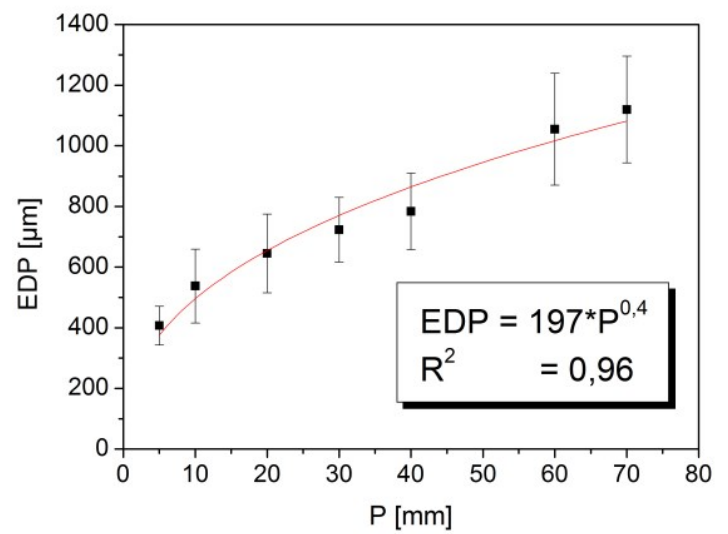

Figura 13: Equação experimental do espaçamento dendrítico primário (EDP) em função da posição (P) da interface metal $/$ molde.

A Figura 14 apresenta a equação experimental que correlaciona os espaçamentos dendríticos primários com a taxa de resfriamento, ajustada para um expoente de -0,55, proposto por Bouchard e Kirkaldy [12], apresentando grande concordancia entre os dados experimentais e a previsão teórica. Apesar da modelagem de Borchard e Kirkaldy ter sido desenvolvida para ligas binárias, alguns autores apresentados na tabela 3 ajustaram os resultados encontrados em experimentos com ligas ternárias a esse modelo, obtendo resultados de ajuste satisfatórios. Pode-se concluir também a relação entre EDP e $T_{R}$, demonstando que, para valores de $\mathrm{T}_{\mathrm{R}}$ cada vez mais altos, os valores de EDP tendem a valores mais baixos.

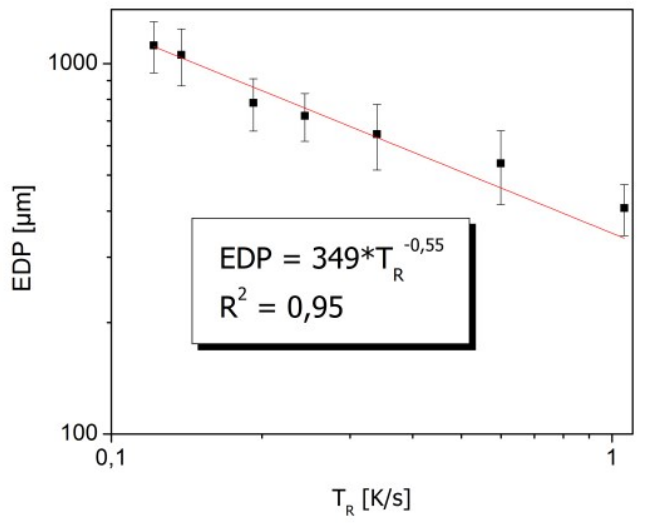

Figura 14: Equação experimental do espaçamento dendrítico primário (EDP) em função da taxa de resfriamento $\left(T_{R}\right)$.

Tabela 3: Leis experimentais para previsão dos espaçamentos dendríticos primários em ligas ternárias.

\begin{tabular}{|l|l|l|}
\hline AUTOR & LIGA & LEI EXPERIMENTAL \\
\hline $\begin{array}{l}\text { Santos [13] } \\
\text { Solidificação Vertical Ascendente }\end{array}$ & Al-3\%Cu-1\%Li & EDP $=220\left(T_{R}\right)^{-0,55}$ \\
\hline $\begin{array}{l}\text { Gomes [1] } \\
\text { Solidificação Vertical Ascendente }\end{array}$ & $\mathrm{Al}-5,5 \% \mathrm{Si}-3 \% \mathrm{Cu}$ & EDP $=216\left(\mathrm{~T}_{\mathrm{R}}\right)^{-0,55}$ \\
\hline $\begin{array}{l}\text { Moutinho [14] } \\
\text { Solidificação Vertical Ascendente }\end{array}$ & $\mathrm{Al}-6 \% \mathrm{Su}-3 \% \mathrm{Cu}$ & $\mathrm{EDP}=200\left(\mathrm{~T}_{\mathrm{R}}\right)^{-0,55}$ \\
\hline Faria [15] & $\mathrm{Al} \% \mathrm{Cu}-4 \% \mathrm{Si}$ & $\mathrm{EDP}=140\left(\mathrm{~T}_{\mathrm{R}}\right)^{-0,55}$ \\
Solidificação Vertical Ascendente & $\mathrm{Al}-4 \% \mathrm{Cu}-4 \% \mathrm{Ag}$ & $\mathrm{EDP}=140\left(\mathrm{~T}_{\mathrm{R}}\right)^{-0,55}$ \\
\hline Bertelli [16] & $\mathrm{Al}-10 \% \mathrm{Sn}-10 \% \mathrm{Cu}$ & $\mathrm{EDP}=235\left(\mathrm{~T}_{\mathrm{R}}\right)^{-0,55}$ \\
\hline
\end{tabular}




\begin{tabular}{|l|l|l|}
\hline Solidificação Vertical Ascendente & Al-20\%Sn-10\%Cu & EDP $=194\left(\mathrm{~T}_{\mathrm{R}}\right)^{-0,55}$ \\
& $\mathrm{Al}-15 \% \mathrm{Sn}-5 \% \mathrm{Si}$ & $\mathrm{EDP}=123\left(\mathrm{~T}_{\mathrm{R}}\right)^{-0,55}$ \\
& $\mathrm{Al}-25 \% \mathrm{Sn}-5 \% \mathrm{Si}$ & $\mathrm{EDP}=204\left(\mathrm{~T}_{\mathrm{R}}\right)^{-0,55}$ \\
\hline Este trabalho & $\mathrm{Al}-10 \% \mathrm{Si}-2 \% \mathrm{Cu}$ & $\mathrm{EDP}=349\left(\mathrm{~T}_{\mathrm{R}}\right)^{-0,55}$ \\
Solidificação Vertical Ascendente & & \\
\hline
\end{tabular}

A Figura 15 apresenta os resultados da variação do EDP em função de $V_{L}$, demonstrando que, para um ajuste de equação de potência para expoente $-1,1$ proposto por Bouchard e Kirkaldy, os dados experimentais não concordam com esse modelamento de ligas binárias. Conclui-se também que, para valores cada vez mais altos de $\mathrm{V}_{\mathrm{L}}$, os valores de EDP tendem a diminuir.

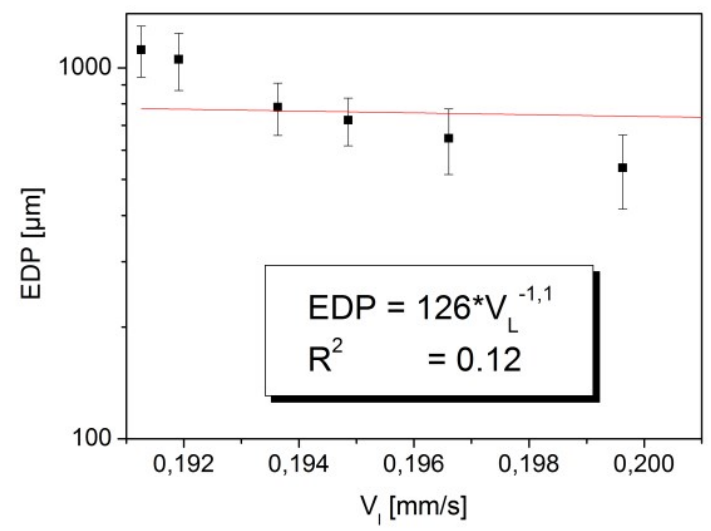

Figura 15: Equação experimental do espaçamento dendrítico primário (EDP) em função da velocidade de deslocamento da isoterma liquidus $\left(\mathrm{V}_{\mathrm{L}}\right)$.

As Figuras 16 (a) e (b) apresentam resultados do Limite de Resistência à Tração (LRT) e do Limite de Escoamento (LE) obtidos no ensaio de tração realizado nos corpos de provas em diversas posições em relação à interface metal $/$ molde.

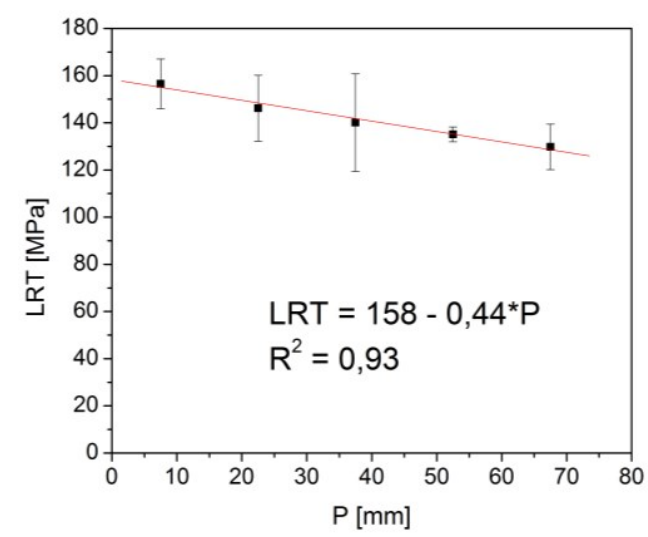

(a)

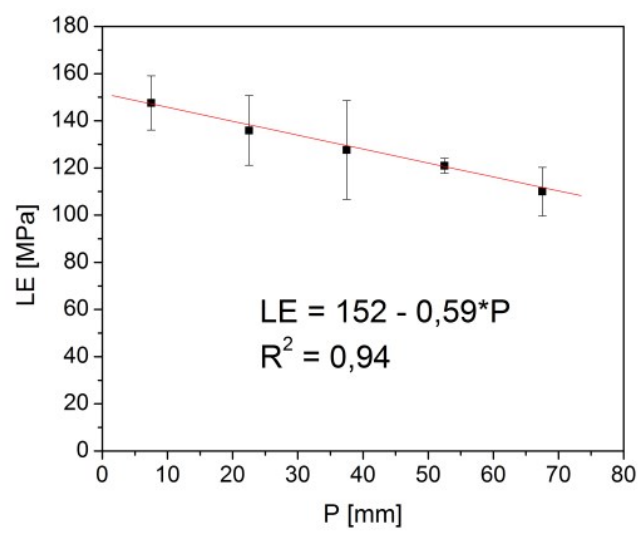

(b)

Figura 16: Resultados do Limite de Resistência à Tração (LRT) e Limite de Escoamento (LE) para as posições específicas em relação a interface Metal/Molde.

Por meio da equação experimental EDP $=197 *(\mathrm{P})^{0,4}$ da figura 11, obteve-se o valor de EDP para cada uma das posições de referência dos corpos de prova, correlacionando-se dessa forma os valores de LRT = 
$\mathrm{f}(\mathrm{EDP})$ e $\mathrm{LE}=\mathrm{f}(\mathrm{EDP})$. Foi utilizada a equação do tipo Hall-Petch $\left(\mathrm{LRT}=\mathrm{LRT}_{0}+\mathrm{K} \cdot \mathrm{EDP}^{-0,5} \mathrm{e} \mathrm{LE}=\mathrm{LE}_{0}+\right.$ K.EDP ${ }^{-0,5}$ ) para obter as equações experimentais, determinado os coeficientes $\mathrm{LRT}_{0}$, $\mathrm{LE}_{0}$ e $\mathrm{K}$ pelo Método dos Mínimos Quadrados. Os dados dessa correlação são apresentados na Figura 17.

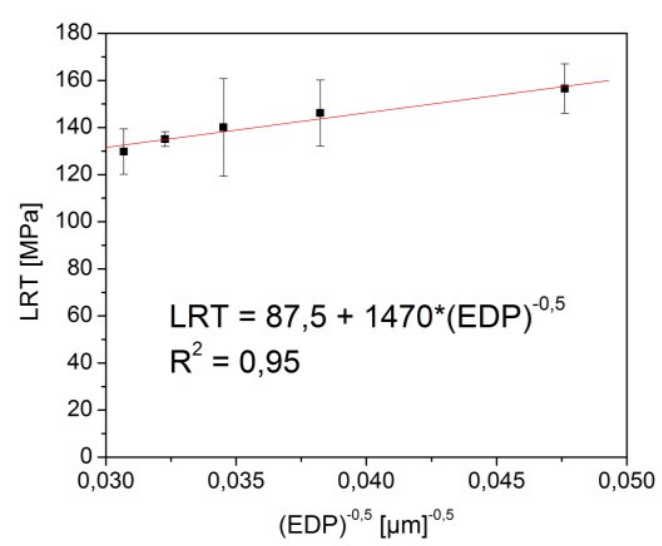

(a)

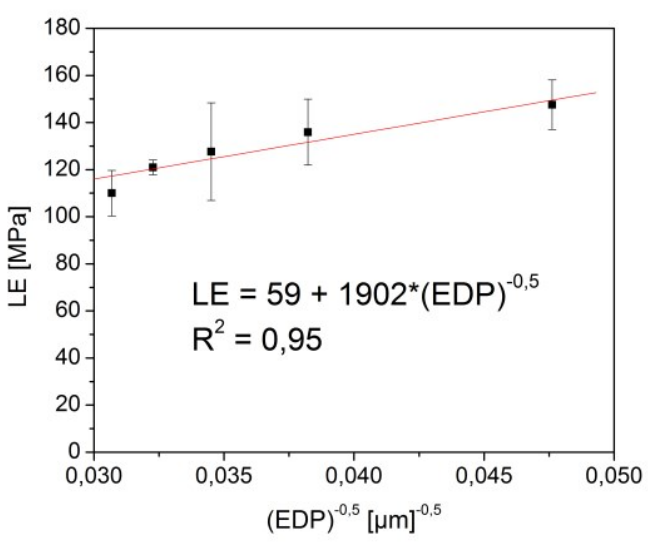

(b)

Figura 17: (a) Correlação entre o limite de resistência à tração (LRT) e espaçamento dendrítico primário (EDP). (b) Correlação entre o limite de escoamento (LE) e espaçamento dendrítico primário (EDP).

As equações experimentais para LRT e LE em função do EDP permitem concluir que, para espaçamentos dendríticos primários com valores cada vez maiores, os valores de LRT e LE tendem aos valores de $\mathrm{LRT}_{0}$ e LE $\mathrm{L}_{0}$, ou seja, estrutura mais refinada permitem valores maiores dessas propriedades mecânicas. Outros autores apresentados na Tabela 4 realizaram estudos nesse sentido reforçando essa correlação entre essas propriedades e espaçamento dendrítico primário.

Tabela 4: Equações experimentais para LRT em função do EDP.

\begin{tabular}{|c|c|c|c|}
\hline AUTOR & LIGA & TIPO ESTRUTURA & EQUAÇÃO EXPERIMENTAL \\
\hline Santos [13] & $\begin{array}{l}\mathrm{Al}-3 \% \mathrm{Cu}-1 \% \mathrm{Li} \\
\mathrm{Al}-6 \% \mathrm{Zn}\end{array}$ & Colunar Celular & $\begin{array}{l}\mathrm{LRT}=120+1100 \cdot \mathrm{EDP}^{-5} \\
\mathrm{LRT}=18+210 . \mathrm{EDP}^{-0,5}\end{array}$ \\
\hline Goulart [17] & $\begin{array}{l}\mathrm{Al}-0,5 \% \mathrm{Fe} \\
\mathrm{Al}-1,0 \% \mathrm{Fe} \\
\mathrm{Al}-1,5 \% \mathrm{Fe}\end{array}$ & Colunar Celular & $\begin{array}{l}\mathrm{LRT}=62,8+98,3 \cdot \mathrm{EDP}^{-0,5} \\
\mathrm{LRT}=60,2+90,5 . \mathrm{EDP}^{-0,5} \\
\mathrm{LRT}=57,6+65,4 . \mathrm{EDP}^{-0,5}\end{array}$ \\
\hline Brito [18] & $\mathrm{Al}-3 \% \mathrm{Mg}-1 \% \mathrm{Si}$ & Colunar Celular & $\mathrm{LRT}=103+252 . \mathrm{EDP}^{-0,5}$ \\
\hline Este trabalho & $\mathrm{Al}-10 \% \mathrm{Si}-2 \% \mathrm{Cu}$ & Colunar Dendrítico & $\mathrm{LRT}=87,5+1470 \cdot \mathrm{EDP}^{-0,5}$ \\
\hline
\end{tabular}

\section{CONCLUSÕES}

Os resultados experimentais obtidos e analisados neste trabalho permitem que sejam extraídas as seguintes conclusões:

- Os valores experimentais de $V_{L}$ e $T_{R}$ diminuem com o avanço da isoterma liquidus, isto é, quanto mais próximo da base refrigerada maiores são esses parâmetros. Os mesmos podem ser representados por em função da posição dadas por $\mathrm{V}_{\mathrm{L}}=0,21(\mathrm{P})^{-0,022}$ e $\mathrm{T}_{\mathrm{R}}=3,96(\mathrm{P})^{-0,82}$.

- Os espaçamentos dendríticos primários aumentam progressivamente da interface metal/molde para o interior do lingote. Isso ocorre porque, durante a solidificação, ocorre o aumento da resistência térmica promovida pela formação do metal sólido.

- Para a liga estudada, solidificada nas condições impostas neste trabalho, a evolução dos espaçamentos dendríticos primários pode ser representada por uma lei experimental dada pela forma geral $\operatorname{EDP}=349\left(T_{R}\right)^{-0,55}$, demonstrando que, para valores maiores de $\mathrm{T}_{\mathrm{R}} \mathrm{o}$ EDP tende a ter valores menores. 
- O Limite de Resistência à Tração (LRT) e o Limite de Escoamento (LE) se correlacionam com os valores de EDP por leis experimentais dadas por $\mathrm{LRT}=87,5+1470 \cdot \mathrm{EDP}^{-0,5} \mathrm{e} \mathrm{LE}=59+1902 \cdot \mathrm{EDP}^{-0,5}$, comprovando a relação entre EDP com as propriedades mecânicas LRT e LE.

- A equação experimental que relaciona o LRT com $T_{R}$ tem apresenta boa aproximação para o modelo de ligas binárias propostos por Bouchard e Kirkaldy mesmo que a liga em estudo seja ternária. Outros trabalhos apresentados com ligas ternárias de alumínio apresentam o mesmo resultado.

- As equações experimentais que correlacionam LRT em função de EDP e LE em função de EDP permitem concluir que, para valores cada vez maiores de EDP os valores de LRT e LE tendem a diminuir a um valor mínimo. Isso permite concluir que, para estruturas mais refinadas as propriedades mecânicas citadas tendem a ter valores maiores. Outros trabalhos apresentados pela literatura com diversas ligas de alumínio apresentam a mesma conclusão.

\section{AGRADECIMENTOS}

Agradecemos o apoio da CAPES mediante o programa pró-equipamentos, que permitiu adquirir os microscópios necessários para a realização deste trabalho.

\section{BIBLIOGRAFIA}

[1] GOMES, L.G; "Microestrutura dendrítica, macrossegregação e microporosidade na solidificação de ligas ternárias Al-Si-Cu", Tese de D. Sc., UNICAMP, Campinas, 2012.

[2] GOMES, C. H. U. et al. "On the Natural Convection in the Columnar to Equiaxed Transition in Directionally Solidified Aluminum-based Binary and Multicomponent Alloys", Mat. Res., v. 18, n. 6, pp. 13621371, dec. 2015.

[3] OSÓRIO, W.R.R., et. al. "Electrochemical corrosion parameters of as-cast $\mathrm{Al} \mathrm{Fe}$ alloys in a $\mathrm{NaCl}$ solution", Corrosion Science, v. 52, pp. 2979-2993, sep. 2010.

[4] SONG, G., BOWLES, A.L., ST JOHN, D.H., "Corrosion Resistance of Aged Die Cast Magnesium Alloy AZ 91D”, Materials Science Engineering, v. 366A, pp. 74-86, Feb. 2004.

[5] ASTA, M., et al., "Solidification Microstructures and Solid-State Parallels: Recent Developments, Future Directions", Acta Materialia v. 57, pp. 941-971, feb. 2009.

[6] BOETTINGER, W. J., et al. "Solidification Microstructures: Recent Developments, Future Directions", Acta Materialia, v.48, pp. 43-70, jan. 2000.

[7] GARCIA, A. - Solidificação: Fundamentos e Aplicações, $2^{\circ}$ ed, Campinas. Editora da Universidade Estadual de Campinas, Unicamp, 2007.

[8] ROSA, D. M., SPINELLI, J. E., GARCIA, A. "Tertiary Dendrite Arm Spacing During Downward Transient Solidification of Al-Si Alloys", Materials Letters, v. 60, pp. 1871-1874, jul. 2006.

[9] KURZ, W, R TRIVERDI. "Solidification microstructures: Recent developments and future directions", Acta Metallurgica et Materialia, v. 38, pp 1-17, jan. 1990.

[10] GUNDUZ, M., ÇARDILI, E. "Directional Solidification of Aluminium-Copper Alloys", Materials Scence and Engineering A, v. 327, pp. 167-185, apr. 2002.

[11] DIAS FILHO, J. M., et al.,"Influência das Variáveis Térmicas Sobre os Espaçamentos Dendríticos Terciários durante a Solidificação Direcional Horizontal da Liga Al-6\%Cu", Matéria (Rio J.), v. 20, n. 1, pp.4763, mar. 2015.

[12] BOUCHARD, D. and KIRKALDY, J. S. "Prediction of Dendrite Arm Spacings in Unsteady and Steady-State Heat Flow of Unidirectionally Solidified Binary Alloys", Metallurgical and Materials Transactions $B$, v. 28B, pp. 651-663, aug. 1997.

[13] SANTOS, G. A. Correlação entre Variáveis Térmicas de Solidificação, Microestrutura e Comportamento Mecânico de Ligas Al-Zn e Al-Cu-Si, Tese de D. Sc., Instituto Tecnológico de Aeronáutica, ITA, São José dos Campos (SP), 2009.

[14] MOUTINHO, D. J. C.; “Análise da Evolução Microestrutural e da Formação de Macrossegregação e Microporosidade na Solidificação Unidirecional Transitória de Ligas Ternárias Al-Cu-Si”, Tese de D. Sc., UNICAMP, Campinas, 2012.

[15] FARIA, Jonas Dias et al . "Influência na microestrutura e na microdureza decorrente da adição de 4\%Ag na liga Al-4\%Cu solidificada unidirecionalmente", Matéria (Rio J.), v. 20, n. 4, pp. 992-1007, dec. 2015.

[16] BERTELLI, F., et al. "Cooling thermal parameters, microstructure, segregation and hardness in direc- 
tionally solidified Al-Sn-(Si;Cu) alloys", Materials and Design, v. 72, pp. 31-42, Feb. 2015.

[17] GOULART, P., et al. "The effects of cell spacing and distribution of intermetallic fibers on the mechanical properties of hypoeutectic Al-Fe alloys", Materials Chemistry and Physics, v. 119, pp. 272-278, jan. 2010.

[18] BRITO, C., et al. "Cellular/dendritic arrays and intermetallic phases affecting corrosion and mechanical resistances of an Al-Mg-Si alloy", Journal of Alloys and Compounds, v. 673, pp. 220-230, jul. 2016. 